\title{
Malformaciones urológicas y síndrome nefrótico: informe de un caso
}

\section{Urological malformations and nephrotic syndrome: A case report}

\author{
Alexandra Palacios-Legarda, ${ }_{1}$ Junior Gahona-Villegas, ${ }^{2}$ Alida Romero, ${ }^{3}$ Silvestre García-de la Puente ${ }^{4}$
}

\begin{abstract}
Resumen
ANTECEDENTES: El síndrome nefrótico supone una incidencia de 1-3 por cada 100,000 niños menores de 16 años. Más de $90 \%$ de estos casos se manifiesta entre el primero y los 10 años de edad. El síndrome nefrótico secundario a nefropatía por reflujo es una alteracion reportada en adultos; sin embargo, no se ha establecido claramente en niños. CASO CLÍNICO: Niña de 2 años y 5 meses, con diagnóstico previo de riñón izquierdo con doble sistema pieloureteral completo, uréter proveniente del polo superior ectópico y aparente reflujo. Ingresó al servicio de Nefrología pediátrica por un cuadro de 15 días de evolución, caracterizado por edema palpebral bilateral, que se irradiaba a los miembros inferiores, de predominio matutino, hipertensión y hematuria. Los estudios de laboratorio reportaron: dislipidemia, proteinuria en los límites nefróticos, hematuria, hipoalbuminemia, complemento normal y marcadores virales negativos. Se prescribieron corticosteroides a dosis plena $\left(60 \mathrm{mg} / \mathrm{m}^{2} / \mathrm{día}\right)$, con lo que disminuyó el edema. Sin embargo, persistieron la proteinuria, hematuria e hipertensión. Se estableció el diagnóstico de corticorresistencia. La biopsia renal evidenció glomeruloesclerosis focal y segmentaria tipo celular. El estudio genético fue negativo para identificar algún gen relacionado con el síndrome nefrótico. Al momento, la paciente se encuentra en seguimiento en el servicio de Nefrología pediátrica. Con el reporte histopatológico se inició el tratamiento con un inhibidor de calcineurina y corticoides a dosis bajas, con mejoría en los estudios paraclínicos. Hoy día padece síndrome nefrótico con remisión parcial.

CONCLUSIONES: Es importante continuar con el reporte de este tipo de casos, con la finalidad de establecer el tratamiento multidisciplinario e integral adecuado para niños. PALABRAS CLAVE: Síndrome nefrótico; anomalías congénitas del riñón y de la vía urinaria; edema; hipertensión; niños.
\end{abstract}

Abstract

BACKGROUND: Nephrotic syndrome assumes an incidence of 1-3 per 100,000 children under 16 years. More than $90 \%$ of these cases manifest between the first and 10 years of age. Nephrotic syndrome secondary to reflux nephropathy is an alteration reported in adults; however, it has not been clearly established in children.

CLINICAL CASE: 2-year-old and 5-month-old girl, with a previous diagnosis of the left kidney with a double full pyeloureteral system, ureter from the ectopic upper pole and apparent reflux. He was admitted to the Pediatric Nephrology service for a 15-day course of evolution, characterized by bilateral eyelid edema, which radiated to the lower limbs, predominantly in the morning, hypertension and hematuria. Laboratory studies reported: dyslipidemia, proteinuria in the nephrotic limits, hematuria, hypoalbuminemia, normal complement and negative viral markers. Full dose corticosteroids (60 $\mathrm{mg} / \mathrm{m}^{2} /$ day) were prescribed, which reduced edema. However, proteinuria, hematuria

\footnotetext{
${ }^{1}$ Residente de tercer año de Pediatría, Pontificia Universidad Católica de Ecuador.

${ }^{2}$ Nefrólogo pediatra, Hospital Pediátrico Baca Ortiz; docente de posgrado de Pediatría, Pontificia Universidad Católica del Ecuador

${ }^{3}$ Nefróloga pediatra, jefa del servicio de Nefrología, Hospital Pediátrico Baca Ortiz; docente de posgrado de Pediatría, Pontificia Universidad Católica del Ecuador.

${ }^{4}$ Departamento de Metodología de la Investigación, Instituto Nacional de Pediatría, Ciudad de México.
}

Recibido: 27 de febrero 2019

Aceptado: 15 de octubre 2019

Correspondencia

Alexandra Palacios-Legarda

alexandra_2910@hotmail.com

Este artículo debe citarse como Palacios-Legarda A, Gahona- J, Romero- A, García-de la Puente S. Malformaciones urológicas y síndrome nefrótico informe de un caso. Acta Pediatr Mex. 2019;40(6):328-34. 
and hypertension persisted. The diagnosis of corticorresistencia was established. Renal biopsy showed focal and segmental glomerulosclerosis cell type. The genetic study was negative to identify any gene related to the nephrotic syndrome. At the moment, the patient is being monitored by the Pediatric Nephrology service. With the histopathological report, treatment was initiated with calcineurin inhibitor and corticosteroids at low doses, with improvement in paraclinical studies. Today he suffers from nephrotic syndrome with partial remission.

CONCLUSIONS: It is important to continue reporting these types of cases, in order to establish the appropriate multidisciplinary and comprehensive treatment in children. KEYWORDS: Nephrotic syndrome; Segmental glomeruloesclerosis; Nephrourological congenital anomalies; Edema; Hypertension; Child.

\section{ANTECEDENTES}

El síndrome nefrótico, según la Kidney Disease: Improving Global Outcomes (K-DIGO), supone una incidencia de 1-3 casos por cada 100,000 niños menores de 16 años. Más de $90 \%$ de estos casos se manifiesta entre el primero y los 10 años de edad. ${ }^{1}$ Su prevalencia, según los registros estadounidenses, se encuentra cercana a los 16 casos por cada 100,000 niños, ${ }^{2}$ datos similares los reporta la Sociedad Española de Nefrología Pediátrica. Alrededor de $10 \%$ de los casos es resistente a los corticosteroides, incluso representa dificultad para el tratamiento. ${ }^{3}$ El síndrome nefrótico corticorresistente, específicamente la glomeruloesclerosis focal y segmentaria, se asocia con $50 \%$ de riesgo de enfermedad renal crónica durante los cinco años del diagnóstico, siempre y cuando no haya remisión parcial o completa; por tanto, los niños con esta enfermedad tienen reducida expectativa de vida. ${ }^{4}$

El síndrome nefrótico en pacientes pediátricos se define por: proteinuria superior a $40 \mathrm{mg} / \mathrm{m}^{2} / \mathrm{h}$ ( 50 mg/kg/día) o cociente proteína-creatinina en orina mayor de $2 \mathrm{mg}$, hipoalbuminemia menor de $2.5 \mathrm{~g} / \mathrm{dL}$ y coexistencia de edema e hiperlipemia. ${ }^{5}$ El síndrome nefrótico en niños se clasifica en tres categorías: primario o idiopático, que a su vez se subdivide en relación con los hallazgos histopatológicos en: enfermedad de cambios mínimos, glomeruloesclerosis focal y segmentaria, glomerulonefritis membranoproliferativa, ne- fropatía membranosa y proliferación mesangial difusa, con mutaciones en más de una docena de genes en el síndrome nefrótico corticorresistente no sindrómico, por ejemplo: nefrina (NPHS1), podocina (NPHS2) y la proteína asociada con CD2 (CD2AP), que son los principales elementos estructurales del diafragma de filtración; así también se han determinado NPHS3 [PLCE1], ACTN4, CD2AP, TRPC6, INF2, PTPRO, ITGA3, MYO1E, ARHGDIA, COQ2, COQ6, ADCK4). Además, las manifestaciones del síndrome nefrótico corticorresistente pueden deberse a mutaciones en genes que codifican: factores de transcripción (WT1, LMX1B); componentes de la membrana basal glomerular (LAMB2, ITGB4); proteínas lisosomales (SCARB2); algún mediador reestructural del nucleosoma de ADN (SMARCAL1) y proteínas mitocondriales (COQ6). ${ }^{5}$

El síndrome nefrótico secundario se relaciona con una enfermedad sistémica identificable, infección que a menudo se acompaña de un componente nefrítico o de tipo adaptativo con respuesta funcional-estructural mediada por hipertrofia glomerular o hiperfiltración asociada con hidronefrosis, agenesia renal y nefropatía por reflujo. La enfermedad congénita se manifiesta en los tres primeros meses de vida. ${ }^{5}$

Las anomalías congénitas del riñón y de la vía urinaria (CAKUT en inglés) representan entre 15 y $20 \%$ de las alteraciones detectadas prenatalmente, con una tasa de 0.3-1.3 por cada 1000 
recién nacidos. Son la primera causa de insuficiencia renal en niños. La prevalencia estimada en Colombia varía de $0.2-0.4 \%{ }^{;}{ }^{6}$ hasta la fecha no hay datos de México. Constituyen una amplia variedad de defectos: obstrucción de la unión ureteropélvica, reflujo vesicoureteral, valvas posteriores, agenesia, displasia o poliquistosis renal. Aunque la etiología de las anomalías congénitas del riñón y de la vía urinaria es multifactorial, existen genes implicados en su expresión. PAX2 fue el primer gen identificado en esta enfermedad, posteriormente se identificaron: AGT R2, BMP4, GDNF, RET, WT1.? En cuanto al doble sistema pielocalicial, es una de las anomalías más frecuentes, donde el uréter que drena la porción superior del riñón (uréter del hemisistema superior) termina de manera más caudal y medial que el que drena su porción inferior (uréter del hemisistema inferior); este desplazamiento del uréter superior de su posición habitual hace más probable que desemboque ectópicamente y se asocie con ureterocele, lo que resulta en obstrucción; por tanto, existe mayor daño estructural. Por su parte, el menor tiempo de desarrollo del uréter inferior disminuye su capa muscular y facilita la aparición de reflujo vesicoureteral. ${ }^{7}$

\section{CASO CLÍNICO}

Niña de 2 años y 5 meses, sin antecedentes familiares ni perinatales de importancia para el padecimiento actual, con diagnóstico previo de infección de vías urinarias a los 6 meses de edad, por lo que se realizó ecografía renal y de vías urinarias con hallazgo de riñón izquierdo con doble sistema pieloureteral completo, uréter proveniente del polo superior ectópico y aparente reflujo. El estudio se complementó con uretrocistografía miccional, que reportó reflujo vesicoureteral grado $\mathrm{V}$, con dilatación del uréter, de apariencia tortuosa, de $20 \mathrm{~mm}$. El urograma excretor evidenció el polo superior renal izquierdo sin función; por tanto, fue necesario realizar gammagrafía renal MAG 3, que demostró hidro- nefrosis con deterioro funcional bilateral. La cistoscopia reportó el meato ureteral derecho sin alteraciones; al meato ureteral izquierdo del segmento inferior y superior ectópico, amplio y refluyente. Con estos hallazgos se decidió efectuar, al año de edad, ureteronastomosis lateroterminal izquierda.

La paciente ingresó al servicio de Nefrología pediátrica por un cuadro de 15 días de evolución, caracterizado por edema palpebral bilateral, que se irradiaba a los miembros inferiores, de predominio matutino, hipertensión y hematuria. Los estudios de laboratorio reportaron: dislipidemia, proteinuria en los límites nefróticos, hematuria, hipoalbuminemia, complemento normal y marcadores virales negativos, por lo que se estableció el diagnóstico de síndrome nefrótico de inicio temprano, asociado con hipertensión y hematuria. Se prescribió tratamiento de soporte con corticoides a dosis plena (60 mg/m²/día), con lo que disminuyó el edema. Sin embargo, en los estudios de control persistieron la proteinuria, hematuria e hipertensión. Se estableció el diagnóstico de corticorresistencia, por lo que se decidió obtener una biopsia renal, con reporte de glomeruloesclerosis focal y segmentaria tipo celular. Al observar esta asociación patológica y basada en los reportes bibliográficos previos se decidió realizar el estudio genético en búsqueda de la causa específica de la alteración; no obstante, resultó negativo para los genes estudiados para síndrome nefrótico.

Al momento, la paciente se encuentra en seguimiento por parte del servicio de Nefrología pediátrica. Con base en el reporte histopatológico se inició el tratamiento con un inhibidor de calcineurina y corticoides a dosis bajas, con mejoría en los estudios paraclínicos. Hoy día padece síndrome nefrótico con remisión parcial. Cuadro 1, Figura 1 
Palacios-Legarda A, et al. Malformaciones urológicas y síndrome nefrótico

Cuadro 1. Exámenes complementarios y tratamiento administrado (continúa en la siguiente página)

\begin{tabular}{|c|c|c|c|c|c|c|}
\hline Valores séricos & Ingreso & $\begin{array}{c}5 \text { días } \\
\text { de corticoide }\end{array}$ & $\begin{array}{c}10 \text { días } \\
\text { de corticoide }\end{array}$ & $\begin{array}{c}\text { Reingreso } \\
\text { para biopsia }\end{array}$ & $\begin{array}{c}\text { Control } \\
\text { a los } 4 \text { meses }\end{array}$ & $\begin{array}{c}\text { Control a los } 10 \\
\text { meses }\end{array}$ \\
\hline Urea & 18 & 30.2 & 24.8 & 26 & 10.2 & 18.5 \\
\hline Creatinina & 0.1 & 0.1 & 0.1 & 0.1 & 0.1 & 0.1 \\
\hline Proteínas totales & 4.2 & 4.7 & 4 & 5.4 & 5.5 & 5.06 \\
\hline Albúmina & 2 & 2.37 & 1.9 & 2.3 & 3.2 & 2.62 \\
\hline Sodio & 140 & 134 & 134 & 128 & 136 & 140 \\
\hline Potasio & 4.7 & 4.1 & 3.7 & 5.3 & 3.9 & 4.28 \\
\hline Cloro & 109 & 95 & 103 & 104 & 99.9 & 106 \\
\hline Calcio & & 8.4 & $8(9.2)$ & & 9.08 & $8.45(9.5)$ \\
\hline Magnesio & & 1.86 & 2 & & 1.9 & 2.06 \\
\hline Fósforo & & & 4.66 & 5.2 & 5.04 & 4.89 \\
\hline Ácido úrico & & & 3.2 & & & 2.9 \\
\hline Leucocitos & 6900 & 13,800 & 17,800 & 10,200 & 9700 & 8400 \\
\hline Neutrófilos & $19.80 \%$ & $33.90 \%$ & $35 \%$ & $37 \%$ & $62.90 \%$ & $57.50 \%$ \\
\hline Linfocitos & $64.20 \%$ & $52 \%$ & $54 \%$ & $52 \%$ & $30.20 \%$ & $33 \%$ \\
\hline Hemoglobina & 10.9 & 2.4 & 11.3 & 12.7 & 12 & 13.1 \\
\hline Hematocrito & 33.6 & 38.4 & 35.8 & 40.6 & 36.4 & 40.3 \\
\hline Plaquetas & 435,000 & 764,000 & 864,000 & 560,000 & 487,000 & 389,000 \\
\hline Colesterol & 316 & & 278 & 298.1 & & \\
\hline Triglicéridos & 117 & & 317 & 120 & & \\
\hline $\mathrm{C} 3$ & 135 & & & 200 & & 147 \\
\hline C4 & 32.9 & & & 47 & & 31 \\
\hline STORCH & Negativo & & & & & \\
\hline Antiestreptolisina & $<200$ & & & & & \\
\hline Fibrinógeno & 783 & & & & & \\
\hline Dímero D & 486.4 & & & & & \\
\hline $\lg G$ & & & & 520 & & \\
\hline $\lg M$ & & & & 238 & & \\
\hline $\lg A$ & & & & 145 & & \\
\hline \multicolumn{7}{|l|}{ Orina } \\
\hline Densidad & 1016 & 1027 & 1015 & 1022 & 1015 & 1027 \\
\hline Proteínas & +++ & ++++ & +++ & +++ & ++ & ++ \\
\hline Sangre & +++ & +++ & +++ & +++ & - & - \\
\hline Hematíes & 9 & & & & 1 & 2 \\
\hline Bacterias & Escasas & & & & Escasa & Escasa \\
\hline IPC & & 193 & 7.77 & 3.99 & 1.09 & 1.2 \\
\hline Creatinina & & 8.3 & & & 68.5 & 37.2 \\
\hline $\begin{array}{l}\text { Proteínas } 24 \\
\text { horas }\end{array}$ & & $168 \mathrm{mg} / \mathrm{m}^{2} / \mathrm{h}$ & $80.5 \mathrm{mg} / \mathrm{m}^{2} / \mathrm{h}$ & $70.3 \mathrm{mg} / \mathrm{m}^{2} / \mathrm{h}$ & $15.4 \mathrm{mg} / \mathrm{m}^{2} / \mathrm{h}$ & $22.2 \mathrm{mg} / \mathrm{m}^{2} / \mathrm{h}$ \\
\hline
\end{tabular}


Cuadro 1. Exámenes complementarios y tratamiento administrado (continuación)

\begin{tabular}{|c|c|c|c|c|c|c|}
\hline Valores séricos & Ingreso & $\begin{array}{c}5 \text { días } \\
\text { de corticoide }\end{array}$ & $\begin{array}{c}10 \text { días } \\
\text { de corticoide }\end{array}$ & $\begin{array}{c}\text { Reingreso } \\
\text { para biopsia }\end{array}$ & $\begin{array}{c}\text { Control } \\
\text { a los } 4 \text { meses }\end{array}$ & $\begin{array}{c}\text { Control a los } 10 \\
\text { meses }\end{array}$ \\
\hline $\begin{array}{l}\text { Hematíes } \\
\text { dismórficos }\end{array}$ & & $0 \%$ & & & & \\
\hline \multirow[t]{3}{*}{ Tratamiento } & $\begin{array}{c}\text { Amlodipino } \\
10 \mathrm{mg} / \text { día } \\
\text { por vía oral }\end{array}$ & & & $\begin{array}{l}\text { A las } 8 \text { semanas } \\
\text { de tratamiento }\end{array}$ & $\begin{array}{l}\text { Ciclosporina } \\
5 \mathrm{mg} / \mathrm{kg} / \text { día } \\
\text { En dos dosis }\end{array}$ & \\
\hline & $\begin{array}{c}\text { Furosemida } \\
1 \mathrm{mg} / \mathrm{kg} / \\
\text { dosis cada } \\
8 \text { horas, se } \\
\text { disminuye }\end{array}$ & & & $\begin{array}{l}\text { Prednisona } 35 \\
\mathrm{mg} / \mathrm{m}^{2} / 48 \text { h por } \\
\text { vía subcutánea }\end{array}$ & $\begin{array}{c}\text { Prednisona } 5 \\
\text { mg/día }\end{array}$ & \\
\hline & $\begin{array}{l}\text { Prednisona } \\
60 \mathrm{mg} / \mathrm{m}^{2} / \\
\text { día por vía } \\
\text { subcutánea }\end{array}$ & & & & & \\
\hline
\end{tabular}

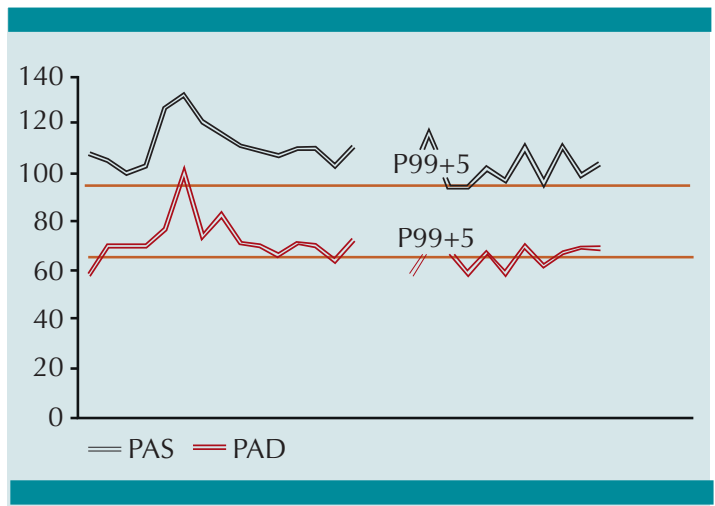

Figura 1. Curva de tensión arterial

\section{DISCUSIÓN}

Las anomalías congénitas del riñón y de la vía urinaria representan un factor predisponente para enfermedad renal crónica, principalmente la duplicación parcial o completa del sistema colector renal, cuya etiología se atribuye a alteraciones genéticas. Según Brenner y Rector (citado por Bakkaloglu et al $)^{5}$ se han identificado más de 30 genes con mutaciones en síndromes mul- tiorgánicos con anomalías congénitas del riñón y de la vía urinaria; sin embargo, la penetrancia es incompleta, con expresión variable, lo que es habitual en las familias implicadas, es decir, dentro de una familia, en particular, en la que los miembros afectados tienen la misma mutación, el fenotipo renal puede variar desde agenesia o displasia hasta anomalías aisladas del sistema colector. De acuerdo con la Academia Española de Pediatría, la manifestación del síndrome nefrótico tiene, también, una base genética; por tanto, deben estudiarse las anomalías genéticas asociadas. $^{8}$

Aunque existen diversas descripciones de las alteraciones individuales, en la bibliografía consultada se encontraron solo tres estudios relacionados con anomalías congénitas del riñón y de la vía urinaria.

En el primer estudio, Mantan y su grupo ${ }^{3}$ revisaron 10 casos de alteraciones congénitas del riñón y las vías urinarias, y encontraron tres pacientes con la asociación de reflujo vesicoureteral y síndrome nefrótico; de éstos, uno tuvo 
glomeruloesclerosis focal y segmentaria, y los demás agenesia o displasia renal. En conclusión, $70 \%$ de los pacientes tuvieron difícil control de la podocitopatía, y 30\% hipertensión arterial, mucho mayor que en otras poblaciones de estudio que solo tuvieron síndrome nefrótico. Por la enfermedad de base genética de ambos, se recomienda efectuar el estudio en búsqueda de alguna alteración que justifique la manifestación concomitante de las alteraciones.

El estudio de Vats y sus colaboradores ${ }^{14}$ informó la asociación del síndrome nefrótico corticorresistente y anomalías congénitas del riñón y de la vía urinaria, relacionado con pérdida del cromosoma 13q. Los autores señalan que debe sospecharse la coexistencia de más de un gen en esta región, encargado del desarrollo y función renal. ${ }^{9}$

Tabares y sus coautores ${ }^{10}$ reportaron un caso de malformación nefrológica relacionado con ectopia renal cruzada concomitante con glomeruloesclerosis focal y segmentaria en un paciente de 40 años. Lo trascendente del caso fue que, por tratarse de corticorresistencia, se descarta la posibilidad de una causa secundaria y el síndrome nefrótico tendría características de ser primario, que apareció con la alteración anatómica.

En cuanto al síndrome nefrótico secundario a nefropatía por reflujo, no puede atribuirse a que ésta sea la causa en la paciente, pues al manifestar la alteración morfofisiológica en el riñón izquierdo, el cambio adaptativo por disminución de las nefronas con hipertrofia glomerular e hiperfiltración, debido a la afectación del segmento superior renal, debería encontrarse daño podocitario en el riñón afectado y no dañar el contralateral, quedando además descartadas otras causas secundarias, ya sean alteraciones inmunológicas o procesos infecciosos. ${ }^{11}$

Con estos datos no puede establecerse una relación causal entre ambas alteraciones; sin em- bargo, por tener un mismo origen embriológico (tanto los riñones como los uréteres provenientes de la yema ureteral primitiva) y sabiendo que las anomalías presentan una base genética, se llevó a cabo el estudio correspondiente, pero al resultar negativo para los genes valorados se excluyó la enfermedad; no obstante, el análisis genético sigue siendo parte importante del estudio. Se tiene como precedente la investigación de Adeyemo y sus colaboradores, ${ }^{12}$ quienes describen HLA-DQA1 y APOL1 como loci de riesgo para el síndrome nefrótico sensible y resistente a los corticoesteroides de inicio en la niñez, lo que sugiere que la genética es la medicina del futuro.

Es importante establecer en el diagnóstico diferencial del síndrome óculo-cerebro-renal de Lowe (OCRL), un trastorno multisistémico caracterizado por cataratas congénitas, glaucoma, capacidad intelectual disminuida, retraso del crecimiento posnatal y disfunción de los túbulos renales con insuficiencia renal crónica, pues muestra una asociación entre malformaciones renales y síndrome nefrótico, pero se descarta por tratarse de una alteración relacionada con el gen OCRL, ligado al cromosoma X, con afectación principal en hombres y asociado con problemas oculares, que la paciente de este estudio no manifestó.

\section{CONCLUSIONES}

Los pacientes con síndrome nefrótico deben valorarse ecográficamente en busca de malformaciones asociadas, pues de acuerdo con los casos reportados, la mayoría expresa glomeruloesclerosis focal y segmentaria, misma que tiene mayor predisposición a la resistencia al tratamiento con corticosteroides. Puesto que ambos representan factores desencadenantes de enfermedad renal crónica en estadios finales a largo plazo, permitirá determinar el pronóstico y riesgo de recurrencia postrasplante. Al tener ambas patologías una base genética, aún no bien establecida, se recomienda realizar el estudio 
correspondiente, con la intención de identificar las alteraciones genéticas responsables de dicha asociación. Es importante continuar con el reporte de este tipo de casos, con la finalidad de establecer el tratamiento multidisciplinario e integral adecuado para los niños.

\section{REFERENCIAS}

1. Cattran DC, et al. Kidney Disease: Improving Global Outcomes (KDIGO), Glomerulonephritis Work Group. KDIGO Clinical Practice Guideline for Glomerulonephritis. Kidney Inter Suppl 2012;2:139-274. https://doi.org/10.1038/ kisup.2012.9

2. Eddy A, et al. Nephrotic syndrome in childhood. Lancet 2003;362:629-39. 10.1016/S0140-6736(03)14184-0.

3. Mantan $\mathrm{M}$, et al. Presence of CAKUT: A predictor of difficult-to-control nephrotic syndrome. Hong Kong J Nephrol 2013;15(1):44-47. https://doi.org/10.1016/j. hkjn.2013.03.006.

4. D'Agati VD, et al. Focal segmental glomerulosclerosis. N Eng J Med 2011;365(25):2398-411. DOI: 10.1056/ NEJMra1106556

5. Bakkaloglu SA, et al. Enfermedades del riñon y del tracto urinario en niños. En: Skorecki K, et al editors. Brener y Rector. El Riñón. 10a ed. Madrid: Elsevier, 2018; 23082364. https://books.google.com.mx/books?id=SqJTDw AAQBAJ \&pg $=P A 2308 \& 1 p g=P A 2308 \& d q=S e v c a n+A .+B a$ kkaloglu,Franz+Schaefer.\&source $=b \mid \& o t s=X s 5 v 2 b-n J 6 \&$ sig=ACfU3U3PgoqwNQ7ThwyKpqYJYhI9sJy3IQ\&hl=es419\&sa=X\&ved=2ahUKEwjJ1az-m7_IAhVSMawKHTU3BL kQ6AEwAHoECAkQAQ\#v=onepage $\& q=S e v c a n \% 20 A . \% 20$ Bakkaloglu\%2CFranz\%20Schaefer.\&f=false
6. Mora-Bautista VM. Anomalías congénitas del riñón y del tracto urinario en niños con síndrome de Down. Rev Colomb Nefrol 2018;5(1):54-60. https://revistanefrologia. org/index.php/rcn/article/view/268/html

7. Palacios-Loro ML, et al. Anomalías nefrourológicas congénitas. Una visión para el pediatra. An Pediatr (Barc) 2015;83:442 DOI: 10.1016/j.anpedi.2015.07.035

8. Román-Ortiz E. Síndrome nefrótico pediátrico. Protoc Diagn Ter Pediatr 2014;1:283-301. https://www.aeped. es/sites/default/files/documentos/18_sindrome_nefrotico.pdf

9. Vats AN, et al. Steroid-resistant nephrotic syndrome and congenital anomalies of kidneys: evidence of locus on chromosome 13q. Kidney Int. 2003 Jul;64(1):17-24. DOI: 10.1046/j.1523-1755.2003.00066.x

10. Melo-Tavares $\mathrm{R}$, et al. Association between focal segmental glomerulosclerosis and cross-fused renalectopia: a new cause of secondary focal segmental glomerulosclerosis or a casual association?. World J Nephrol Urol 2015;4(1):183185. http://www.wjnu.org/index.php/wjnu/article/ view/207/172

11. Rosenblum N. Enfermedades del riñon y del tracto urinario en niños.. En: Skorecki K, et al editors. Brener y Rector. El Riñón. 10a ed. Madrid: Elsevier, 2018;2294-2307. https://books.google.com.mx/books?id $=$ SqJTDwAAQBAJ\&pg=PA2308\&lpg=PA2308\&dq=Sevcan+ A.+Bakkaloglu,Franz+Schaefer. \&source $=$ bl\&ots $=X s 5 v 2 b-n$ J6\&sig=ACfU3U3PgoqwNQ7ThwyKpqYJYhI9sJy3IQ\&hl=es419\&sa=X\&ved=2ahUKEwjJ1az-m7_|AhVSMawKHTU3BL kQ6AEwAHoECAkQAQ\#v=onepage\&q=Sevcan\%20A.\%20 Bakkaloglu\%2CFranz\%20Schaefer.\&f=false

12. Adeyemo A, et al. HLA-DQA1 and APOL1 as Risk Loci for Childhood-Onset Steroid-Sensitie and Steroid-Resistant Nephrotic Syndrome. Am J Kidney Dis DOI: 10.1053/j. ajkd.2017.10.013 